\title{
Effects of oat-bran concentrate on rat serum lipids and liver fat infiltration
}

 \\ O. HÄNNINEN² AND K. SYRJÄNEN ${ }^{3}$ \\ ${ }^{1}$ Technical Research Centre of Finland, Food Research Laboratory, PO Box 203, FIN-02151 \\ Espoo, Finland \\ Departments of ${ }^{2}$ Physiology and ${ }^{3}$ Pathology, University of Kuopio, PO Box 1627, FIN-70211 \\ Kuopio, Finland
}

(Received 17 June 1992 - Accepted 4 February 1993)

\begin{abstract}
Oat bran concentrated with respect to both soluble and insoluble dietary fibre was fed to adult rats, and its effects on serum cholesterol and liver fat infiltration were studied. The feeds contained 15,30 or $45 \mathrm{~g}$ $\beta$-glucan/kg, except in control groups where the fibre of the feed was cellulose. Half the experimental groups received the feeds with an addition of $10 \mathrm{~g}$ cholesterol and $2 \mathrm{~g}$ cholic acid/kg in order to create an hypercholesterolaemic condition. In normocholesterolaemic rats the concentrated oat bran did not cause any significant changes in serum cholesterol concentrations, but reduced liver weight compared with control group rats fed on the cellulose-containing diet. In hypercholesterolaemic rats the concentrated oat bran reduced serum total cholesterol and increased high-density-lipoprotein cholesterol concentration. The effect was seen already at the $15 \mathrm{~g} / \mathrm{kg}$ concentration of $\beta$-glucan, the higher doses tested did not significantly improve the effect. Infiltration of fat into liver cells, especially in the periportal areas, was observed only in hypercholesterolaemic animals. The fat infiltration was accentuated in proportion to the amount of oat-bran concentrate fed.
\end{abstract}

Oat bran: Cholesterol: Liver: Rat

In recent years the hypocholesterolaemic effect of oat fibre has been extensively studied both in animal and clinical experiments, and preparations concentrated with respect to the active component, $\beta$-glucan, are being developed, as reviewed by Paton (1991). The majority of the animal studies on the physiological effects of oat fibre have been performed using rats. However, the rat is relatively resistant to the induction of hypercholesterolaemia and atherosclerosis (Carrol \& Feldman, 1989). Compared with man, on a normal diet it has a lower (about $2.5 \mathrm{mmol} / \mathrm{l}$ ) serum total cholesterol concentration and a higher value $(0.40 .6)$ for high-density-lipoprotein (HDL)-cholesterol:total cholesterol. To create a hypercholesterolaemic condition in rats it has been common practice to include lipotropic substances, most often cholesterol and cholic acid or other bile acids, in the feed (Carrol \& Feldman, 1989; Shinnick et al. 1990). Of sixteen recent rat studies on the hypocholesterolaemic effects of oat bran, in four studies only (Illman \& Topping, 1985; Ranhotra et al. 1990; Mongeau et al. 1991; Mälkki et al. 1992) were lipotropic substances not used. Addition of cholesterol and bile acids to the diet creates an artificial state where HDL-cholesterol:total cholesterol is about $0 \cdot 2$ (e.g. Ney et al. 1988). It has resulted also in an accumulation of fat in the liver, independently of addition of insoluble (Lopez-Guisa et al. 1988) or soluble (Shinnick et al. 1990) fibre components. According to Ney et al. (1988), soluble oat fibre tends to normalize lipoprotein composition in cholesterol-fed rats. 
However, in studies in which young rats have been used, hypocholesterolaemic effects of oat bran or its fractions have been observed even without an induced hypercholesterolaemia (Ranhotra et al. 1990; Mongeau et al. 1991; Mälkki et al. 1992). In the long-term experiment of Mongeau et al. (1991), a significant hypocholesterolaemic effect of oat bran was observed at week 8 on the experimental diet without added cholesterol or cholic acid, but not at week 29 , and with male rats only. The hypocholesterolaemic effect and also an elevation of HDL-cholesterol were considerably greater in young rats when the soluble fibre was given as oat-bran concentrates than when given as untreated oat bran (Ranhotra et al. 1990; Mälkki et al. 1992). The relative efficiencies of the preparations were shown to depend on the viscosity and molecular weight of the $\beta$-glucan (Mälkki et al. 1992).

The aim of the present study was to test the effect of oat-bran concentrate in adult rats, both normocholesterolaemic and hypercholesterolaemic, and the effect on fat infiltration of the liver, in order to forecast its potential for hypocholesterolaemic effects and its safety in human nutrition. The dose responses were also investigated.

\section{MATERIALS AND METHODS \\ Oat-bran concentrate}

The oat bran used was a commercial product from Raisio Group, Finland, derived from a mixture of domestically-grown oat varieties and produced by conventional dry milling after a heat treatment at $100^{\circ}$ for $90 \mathrm{~min}$. Oat-bran concentrate was prepared on a pilot scale from oat bran by reducing non-fibre components by wet milling in $70 \%(\mathrm{w} / \mathrm{w})$ ethanol solution (Autio et al. 1992). The oat-bran concentrate thus obtained had the following composition $(\mathrm{g} / \mathrm{kg})$ : protein $(\mathrm{N} \times 5.7) 197$, fat 88 , total carbohydrate 561 , $\beta$-glucan 189, ash 44, moisture 110 .

\section{Experimental design and diets}

Feeds adjusted to constant levels of fat, protein, vitamins and minerals, and with increasing levels of $\beta$-glucan originating from oat-bran concentrate were used. Half the experimental groups received the feeds supplemented with $10 \mathrm{~g}$ cholesterol and $2 \mathrm{~g}$ cholic acid $/ \mathrm{kg}$. The composition of the feeds is presented in Table 1.

\section{Animal experiments}

Adult male Mol:SPRD rats obtained from Møllegaard ApS, Ejby, Denmark were used in the experiment which was performed from 15 December 1990 to 5 January 1991. After arrival the rats were adapted for $9 \mathrm{~d}$ before the start of the experiment. During both the adaptation period and the experiment the rats were housed in stainless-steel cages $(420 \times 250 \times 150 \mathrm{~mm}$; open upwards $)$, two rats in each cage, and fed standard rat feed during adaptation. For the experiment the animals, weighing $241-295 \mathrm{~g}$, were divided into groups of ten with approximately the same weight distribution in each group. The animal room was regulated to a temperature of $20 \pm 1^{\circ}$ and relative humidity of $55 \pm 10 \%$, it had fifteen to twenty air changes/h and a light-dark cycle of 14-10 h. The test feeds, given ad lib in powder form, were adjusted to contain oat-bran concentrate corresponding to 15 , 30 and $45 \mathrm{~g} \beta$-glucan $/ \mathrm{kg}$. During the experiment the identity of the test feeds was unknown to the experimenters. Feed consumption was recorded for the two rats together in each cage twice weekly. The animals were weighed individually once weekly. At the beginning of the experiment and after 1,2 and 3 weeks, blood samples were taken between 08.00 and 12.00 hours from the saphenous vein after $14-20 \mathrm{~h}$ fasting. After the last blood sample the animals were weighed, killed by guillotine decapitation and bled. After weighing, the liver samples were taken, frozen in liquid $\mathrm{N}_{2}$, and used for an histochemical examination. The 
Table 1. Composition of the experimental feeds $(\mathrm{g} / \mathrm{kg}$ diet $)$

\begin{tabular}{lrrrrrrrr}
\hline \hline Feed ... & M & I & K & L & H & N & O & P \\
\hline Oat-bran concentrate* & - & 79 & 159 & 238 & - & 79 & 159 & 238 \\
Cellulose & 72 & - & - & - & 72 & - & - & - \\
Vitamin mixture $\dagger$ & 10 & 10 & 10 & 10 & 10 & 10 & 10 & 10 \\
Mineral mixture & 39 & 39 & 39 & 39 & 39 & 39 & 39 & 39 \\
Soya-bean oil & 50 & 43 & 36 & 29 & 50 & 43 & 36 & 29 \\
Maize starch & 678 & 707 & 653 & 622 & 690 & 695 & 641 & 610 \\
Casein & 140 & 121 & 103 & 62 & 140 & 121 & 103 & 62 \\
Cholesterol & - & - & - & - & 10 & 10 & 10 & 10 \\
Cholic acid & - & - & - & - & 2 & 2 & 2 & 2 \\
Protein & 120 & 120 & 120 & 120 & 120 & 120 & 120 & 120 \\
Fat & 50 & 50 & 50 & 50 & 50 & 50 & 50 & 50 \\
$\beta-$ Glucan & - & 15 & 30 & 45 & - & 15 & 30 & 45 \\
\hline
\end{tabular}

* Composition $(\mathrm{g} / \mathrm{kg})$ : protein $(\mathrm{N} \times 5 \cdot 7)$ 197, fat 88, total carbohydrate $561, \beta$-glucan 189 , ash 44, moisture 110 .

$\uparrow$ Vitamin mixture 76 (American Institute of Nutrition, 1977).

$\$$ Prepared according to National Research Council requirements (NRC, 1987).

research plan was approved by the Ethical Committee for Animal Experiments of the University of Kuopio.

\section{Analytical methods}

The $\beta$-glucan contents of the preparations were analysed according to the method of McCleary \& Glennie-Holmes (1985).

Serum total cholesterol concentrations were analysed using an enzymic test kit (CHODPAP, Catalogue no. 237574; Boehringer Mannheim GmbH, Germany). Cholesterol in the HDL fraction was analysed after precipitation of very-low-density (VLDL) and lowdensity lipoproteins (LDL) with dextran sulphate and $\mathrm{MgCl}_{2}$. Serum triacylglycerol concentrations were assayed using an enzymic test kit (GPO-PAP, Catalogue no. 701904; Boehringer Mannheim). Serum alanine aminotransferase (EC 2.6.1.2) activities were determined according to the Scandinavian recommendations (The Committee on Enzymes, The Scandinavian Society for Clinical Chemistry and Clinical Physiology, 1974) using the reagents of Medix Biochemica (Kauniainen, Finland).

\section{Histopathology}

Half the liver from each animal was used for histopathological study. The samples were fixed in neutral formaldehyde $(100 \mathrm{ml} / 1)$, embedded in paraffin and processed into $5 \mu \mathrm{m}$ sections for light microscopy according to routine procedures. The sections were stained with haematoxylin-eosin for histopathological assessment.

The sections were viewed by a single pathologist who was unaware of the diets fed to the animals. The general morphology of each liver was assessed first at low-power magnification to provide a general impression of the histological changes to be anticipated. The changes observed were classified as previously described either as a microvesicular fatty change or a macrovesicular fatty change (Craig, 1990). These changes were classified as mild (type I) and severe (type II) fatty changes, and graded according to the share of hepatocytes affected.

\section{Statistics}

Statistical significances of the differences were analysed using StatView $512+{ }^{\mathrm{TM}}$ program (BrainPower, Inc., Calabasas, CA, USA) on an Apple Macintosh computer. Data for each group at different time-points were analysed by single-factor analysis of variance for 


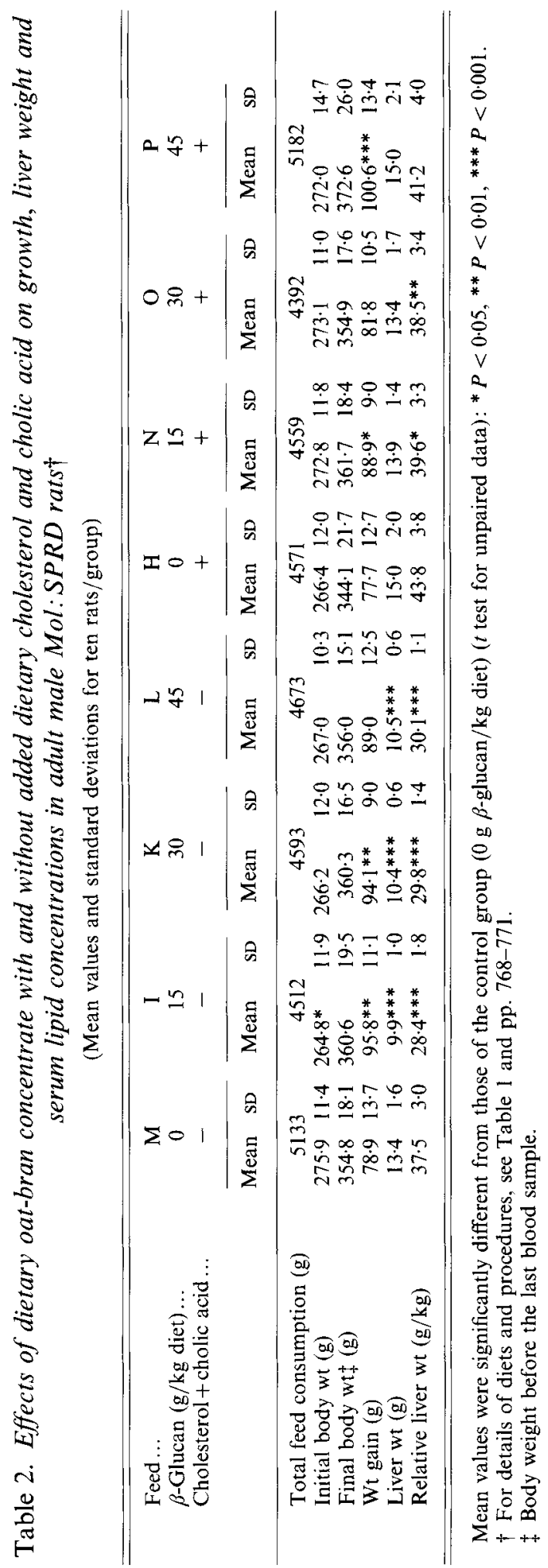




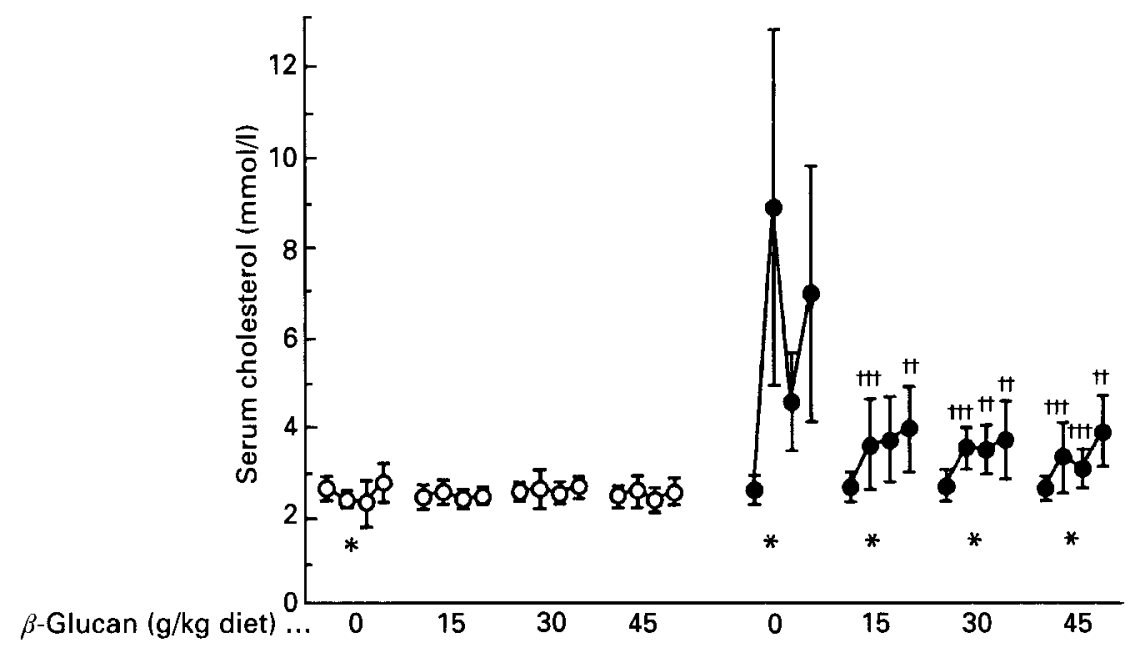

Fig. 1. Effect of dietary oat-bran concentrate $(0,15,30$ and $45 \mathrm{~g} \beta$-glucan $/ \mathrm{kg})$ on serum total cholesterol concentration in rats fed on diets with $(O)$ and without $(O)$ added cholesterol and cholic acid. The four consecutive points in each group represent the values at the start and at weeks 1,2 and 3 of the experiment. Values are means and standard deviations represented by vertical bars for ten rats/group. Mean values for different timepoints within each group were significantly different (ANOVA): ${ }^{*} P<0.05$. Mean values were significantly different from those for the control group ( $t$ test for unpaired values): $\dagger+<0 \cdot 01, \dagger+P<0.001$.

repeated measures (ANOVA). Differences between the control (cellulose) and oat-fibre groups and between the two control groups (with and without lipotropic substances) were tested by a two-tailed $t$ test for unpaired data. Values of $P<0.05$ were considered statistically significant.

\section{RESULTS}

In rats without added lipotropic substances in the diet, oat-bran concentrate increased weight gain and reduced relative liver weight compared with the cellulose control group (Table 2). Oat-bran concentrate had no consistent effect on serum total cholesterol or HDL-cholesterol (Figs. 1 and 2). An increase in serum triacylglycerols from the initial values was observed for all groups (Fig. 3), but varied inconsistently with regard to time and dose of oat-bran concentrate.

Comparison of the effects of the control feeds with (feed $\mathrm{H}$ ) and without (feed $\mathrm{M}$ ) lipotropic substances showed that supplementation of the diets with $10 \mathrm{~g}$ cholesterol and $2 \mathrm{~g}$ cholic acid $/ \mathrm{kg}$ did not affect the weight gain, but increased the relative liver weight $(P<0.001, t$ test $)$. It clearly increased serum total cholesterol level after 1,2 and 3 weeks $(P<0.001)$ and decreased serum HDL-cholesterol level after 3 weeks $(P<0.001)$. Serum triacylglycerol level was significantly higher only after 2 weeks $(P<0 \cdot 05)$.

In rats with induced hypercholesterolaemia, oat-bran concentrate also caused a greater weight gain and lower relative liver weights than those in the cellulose control group (Table 2). It significantly attenuated the increase in total cholesterol and decrease in HDLcholesterol caused by the cholesterol and cholic acid feeding (Figs 1 and 2). No significant dose-response effect was observed, i.e. even the lowest concentration tested, corresponding to $15 \mathrm{~g} \beta$-glucan $/ \mathrm{kg}$ in the feed, gave rise to an attenuating effect which was similar to the effects obtained with the higher concentrations of $\beta$-glucan tested, although a slight nonsignificant tendency for a decrease in total cholesterol and an increase in HDL-cholesterol with increasing content of $\beta$-glucan in the feed was observed. Serum triacylglycerols increased in all groups from the initial values before the experimental diets were given (Fig. 


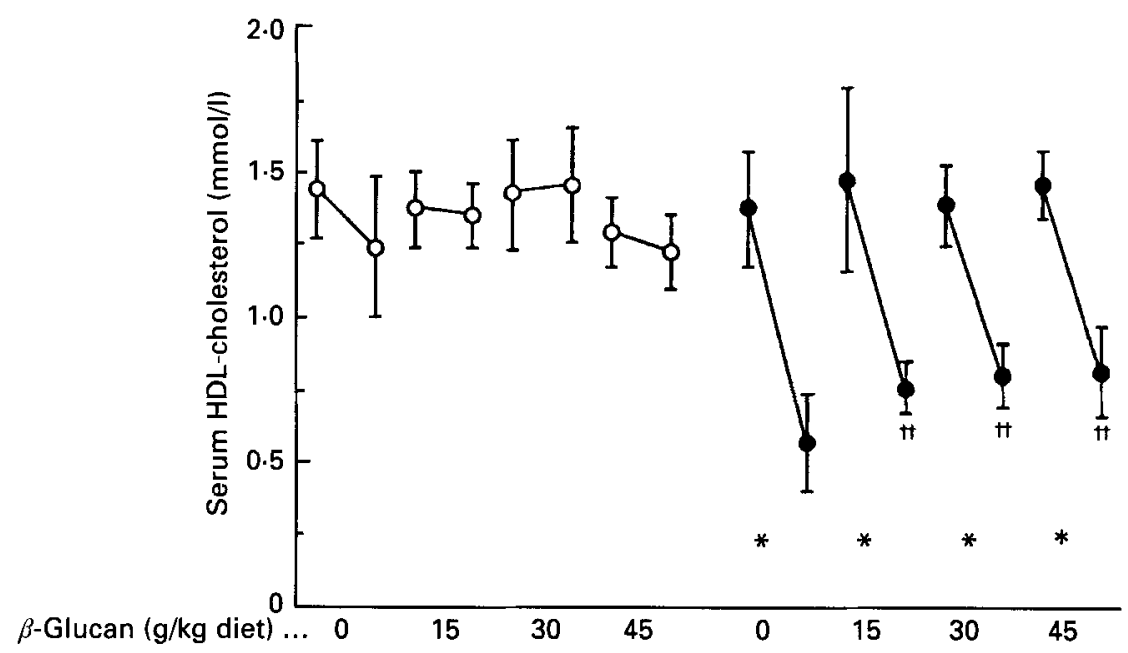

Fig. 2. Effect of dietary oat-bran concentrate $(0,15,30$ and $45 \mathrm{~g} \beta$-glucan $/ \mathrm{kg})$ on serum high-density-lipoprotein (HDL)-cholesterol concentration in rats fed on diets with $(O)$ and without $(O)$ added cholesterol and cholic acid. The two consecutive points in each group represent the values at the start and at week 3 of the experiment. Values are means and standard deviations, represented by vertical bars for ten rats/group. Mean values for different time points within each group were significantly different (ANOVA): ${ }^{*} P<0.05$. Mean values were significantly different from those for the control group ( $t$ test for unpaired values): $\dagger \dagger P<0.01$.

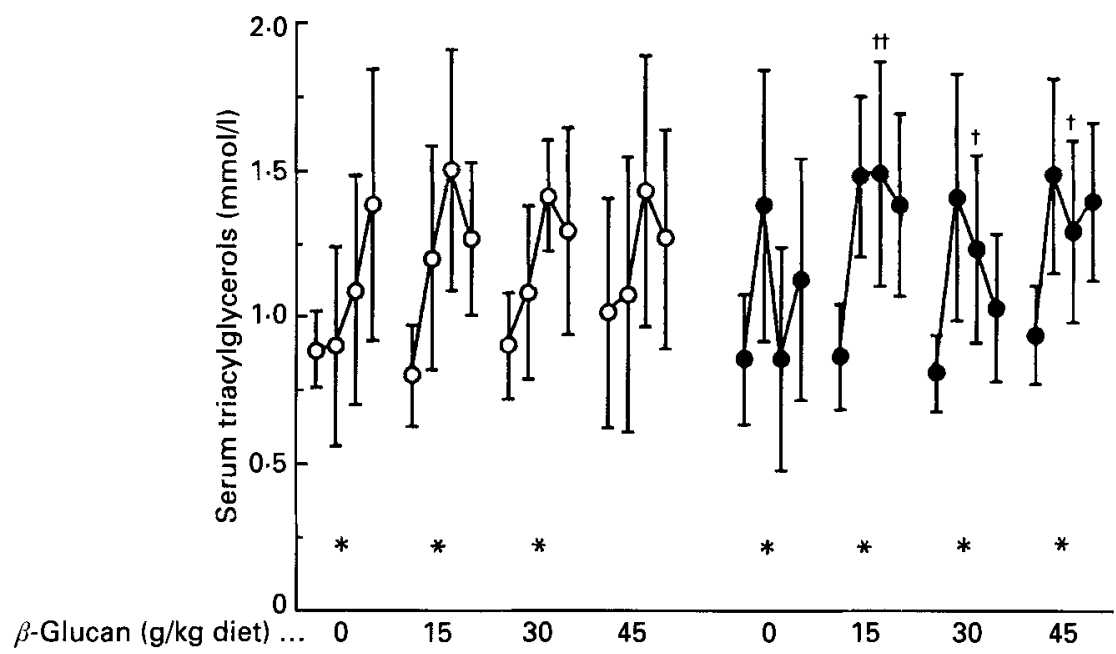

Fig. 3. Effect of dietary oat-bran concentrate $(0,15,30$ and $45 \mathrm{~g} \beta$-glucan $/ \mathrm{kg})$ on serum triacylglycerol concentration in rats fed on diets with (O) and without $(O)$ added cholesterol and cholic acid. The four consecutive points in each group represent the values at the start and at weeks 1,2 and 3 of the experiment. Values are means and standard deviations, represented by vertical bars for ten rats/group. Mean values for different timepoints within each group were significantly different (ANOVA): ${ }^{*} P<0.05$. Mean values were significantly different from those for the control group ( $t$ test for unpaired values): $\uparrow P<0.05$, $\uparrow \dagger P<0.01, \uparrow \dagger \dagger P<0.001$.

3), but there was no difference between the cholesterol and cholic acid-fed and normocholesterolaemic groups, nor between the groups fed on cellulose or oat-bran concentrate.

Lipid infiltration into the liver occurred only in the hypercholesterolaemic rats. The only marked abnormality was the fatty change. In the milder type of changes the fat was in small 
Table 3. Fatty changes in the livers of hypercholesterolaemic rats fed on diets containing different amounts of oat-bran concentrate $(0-45 \mathrm{~g} \beta$-glucan $/ \mathrm{kg})$

(Type I: grade 0 no change, $1+<25 \%$ hepatocytes affected, $2+25-50 \%$ hepatocytes affected, $3+$ $50-75 \%$ hepatocytes affected, $4+75-90 \%$ hepatocytes affected; type II: grade 0 entirely absent, 1 $<10 \%$ periportal areas affected, $210-25 \%$ periportal areas affected, $325-75 \%$ periportal areas affected, $47 \tilde{j}-90 \%$ periportal areas affected)

\begin{tabular}{|c|c|c|c|c|c|c|c|c|c|c|}
\hline \multirow[b]{2}{*}{$\beta$-Glucan $(\mathrm{g} / \mathrm{kg})$} & \multicolumn{5}{|c|}{ Mild changes (type I) } & \multicolumn{5}{|c|}{ Severe changes (type II) } \\
\hline & 0 & $1+$ & $2+$ & $3+$ & $4+$ & 0 & 1 & 2 & 3 & 4 \\
\hline 0 & 0 & 2 & 3 & 2 & 3 & 5 & 2 & 2 & 1 & 0 \\
\hline 15 & 0 & 0 & 3 & 5 & 2 & 0 & 2 & 3 & 4 & 1 \\
\hline 30 & 0 & 0 & 5 & 4 & 1 & 0 & 1 & 3 & 2 & 4 \\
\hline 45 & 0 & 0 & 4 & 6 & 0 & 0 & 0 & 3 & 2 & 5 \\
\hline
\end{tabular}

separate vacuoles of hepatocytes (Plates 1 and 2). The changes were already evident in the hypercholesterolaemic control group fed on cellulose-containing diet. The number of hepatocytes affected increased slightly but not significantly when oat-bran concentrate was included in the diet (Table 3).

More severe fatty changes were observed in the proximity of portal veins. Fat occurred more frequently in separate massive cavities (Plate 3). However, no cirrhotic, necrotic or inflammatory changes were observed. These changes were already present in the hypercholesterolaemic control group, but were accentuated with an increase in oat-bran concentrate in the feed.

Serum alanine aminotransferase activity varied from 42 to 68 units/1, showing no signs of liver cell damage in any of the experimental groups, and no significant difference between the experimental groups.

\section{DISCUSSION}

In our previous study (Mälkki et al. 1992) a 30\% decline in serum total cholesterol concentration was observed in normocholesterolaemic young rats which were fed with a diet containing $33 \mathrm{~g} \beta$-glucan $/ \mathrm{kg}$ originating from an ethanol-water wet-milled oat-bran concentrate. Moreover, compared with cellulose-fed control animals, increased absolute and relative liver weights as well as accumulation of fat in the livers of oat-bran concentrate-fed animals were observed.

In the present study a similar oat-bran concentrate was fed to adult normocholesterolaemic rats in diets containing 15,30 or $45 \mathrm{~g} \beta$-glucan $/ \mathrm{kg}$. In contrast to the young rats used in the previous study, serum total and HDL-cholesterol concentrations were not affected in adult rats. Yet a decrease in the absolute and relative liver weights was observed indicating an interference with the liver metabolism. Lack of effect on serum cholesterol might be partly due to the low initial level of cholesterol in the rats with cholesterol and cholic acid-free diet. No lipid accumulation was observed in the livers of these animals.

The only experimental difference compared with the earlier studies in which a significant reduction was achieved with similar preparations (Ranhotra et al. 1990; Mälkki et al. 1992) was the age of the animals. In the studies of Mongeau et al. (1991) the hypocholesterolaemic effect of oat bran in the absence of added lipotropic substances depended on the age and sex, thus probably also on the sexual maturity of the male animals. There seems to be an inverse correlation between age and hypocholesterolaemic 
response when no lipotropic substances are added in the feed. In the experiments of Ranhotra et al. (1990) the initial weight of Sprague-Dawley rats was 35(SD 3) g, and a concentration of $28.3 \mathrm{~g}$ soluble fibre $/ \mathrm{kg}$ in the feed caused a reduction of $39 \%$ in total cholesterol after 4 weeks, with no significant change in the concentration of serum HDLcholesterol. In our own previous experiments (Mälkki et al. 1992) with Sprague-Dawley rats having an initial weight of 70 (SD 6) g a $\beta$-glucan concentration of $33 \mathrm{~g} / \mathrm{kg}$ caused a decrease of $28 \%$ in serum total cholesterol after 4 weeks. In the study of Illman \& Topping (1985) with Hooded Wistar rats having an initial weight of $200-220 \mathrm{~g}$ a content of $100 \mathrm{~g}$ dietary fibre $/ \mathrm{kg}$, corresponding to approximately $50 \mathrm{~g} \beta$-glucan $/ \mathrm{kg}$, caused a decrease of $15 \%$ in plasma cholesterol within $10 \mathrm{~d}$. In the present study the initial weight of SpragueDawley rats was 270(SD 15) g, and no cholesterol-lowering effect in normocholesterolaemic animals was observed.

The small but significant increase in liver weight in some groups receiving oat-bran preparations observed in our earlier study (Mälkki et al. 1992) was not observed in the present work. On the contrary, both absolute and relative liver weights were lower than in the control groups receiving cellulose in the diet. The main difference in the test conditions was the age of the animals, which might have caused some differences in the liver metabolism. For example, many liver enzyme activities are known to be strongly agerelated in rats (Schmidt et al. 1983). As in the previous study and several other studies, the weight gain of the animals in the test groups receiving oat-bran preparations was higher than that in the control group receiving the cellulose-containing diet.

Shinnick et al. (1990) studied the cholesterol-fed rat as a model for evaluating the hypocholesterolaemic potential of foods. A diet containing $10 \mathrm{~g}$ cholesterol and $2 \mathrm{~g}$ cholic acid $/ \mathrm{kg}$ effectively elevated the concentrations of cholesterol in the serum (by $107 \%$ compared with the control diet, in $20 \mathrm{~d}$ ) and in the liver, and of triacylglycerols in the liver, in adult rats. It also resulted in a $45 \%$ increase in liver weight and severe infiltration of fat in the livers, the severity of the lesions increasing with an increase in cholic acid in the feed.

In the present study rats were made hypercholesterolaemic using a similar procedure. After treatment for 3 weeks, serum total cholesterol level was $150 \%$ higher and HDLcholesterol level 55\% lower than that in the animals fed on the control diet. Relative liver weight increased by $17 \%$ and lipid accumulated in the liver.

With hypercholesterolaemic feeds, fluctuations in the serum total cholesterol and triacylglycerol concentrations during the experiment were large, especially in the control group fed on cellulose-containing diet and in the group receiving the largest concentration of oat-bran concentrate. Since the animals were not adapted to the hypercholesterolaemic conditions before the test, the fluctuation evidently indicates an imbalance in cholesterol metabolism. A similar tendency is evident in the results of Ranhotra et al. (1990) in which the values for total cholesterol in both the control and the experimental groups were 18-28\% higher after 2 weeks than after 4 weeks. In most of the previous studies only values for initial and final concentrations of cholesterol are given.

Oat-bran concentrate in the hypercholesterolaemic diet significantly attenuated the increase in serum total cholesterol and the decrease in HDL-cholesterol level. During treatment for 3 weeks, serum total cholesterol concentration decreased by about $45 \%$ and HDL-cholesterol concentration increased by about $40 \%$, compared with the hypercholesterolaemic control diet. Similar normalization of the serum cholesterol profile of hypercholesterolaemic rats after ingestion of soluble oat-fibre preparations has been reported previously by Ney et al. (1988).

Threshold concentrations and dose responses for the hypocholesterolaemic effect of oatbran preparations in rats were studied recently by Shinnick et al. (1988, 1990). The threshold appeared at $30 \mathrm{~g}$ oat fibre $/ \mathrm{kg}$, corresponding to $15 \beta$-glucan in the feed, although 
the effects were not significant at levels below 40 (Shinnick et al. 1988) or 100 (Shinnick et al. 1990) g oat fibre $/ \mathrm{kg}$. Similarly, Newman et al. (1992) observed a significant reduction in serum cholesterol with oat bran in amounts corresponding to $33.2 \mathrm{~g} \beta$-glucan $/ \mathrm{kg}$ in the feed, but not with an amount corresponding to $13.1 \mathrm{~g} \beta$-glucan $/ \mathrm{kg}$. Liver cholesterol was reduced significantly in the studies of Shinnick et al. $(1988,1990)$ when the content of oat fibre was $4 \%$ or higher, whereas Newman et al. (1992) obtained a reduction with a diet containing $33 \cdot 2 \mathrm{~g}$ dietary fibre from oat bran $/ \mathrm{kg}$ but not with another containing $84 \cdot 2 \mathrm{~g} / \mathrm{kg}$.

In the present study a significant reduction in total cholesterol and an increase in HDLcholesterol, compared with the cellulose-fed control group, was obtained in hypercholesterolaemic rats with a dose corresponding to $15 \mathrm{~g} \beta$-glucan $/ \mathrm{kg}$ in the feed. This is lower than the concentrations reported by the authors cited previously. It confirms that the earlier observations (Ranhotra et al. 1990; Mälkki et al. 1992) on the superiority of the hypocholesterolaemic effect of oat-bran concentrate over untreated oat bran, tested with young rats and on a similar level of $\beta$-glucan either from oat bran or concentrate, are valid also for adult hypercholesterolaemic rats.

Oat-bran concentrate in the hypercholesterolaemic diet decreased the relative liver weight by about $10 \%$, but accentuated the degree of liver fat infiltration in proportion to its concentration. Also in the study of Shinnick et al. (1990), oat fibre in the feed tended to decrease liver weight. All cholesterol and cholic acid-fed animals in their study exhibited moderate to severe fat infiltration. However, no consistent effect of oat fibre on liver fat infiltration was observed.

In the present study an increase in the liver fat accumulation was observed along with the increase in the concentration of fibre in the diet. This might be caused by an increase in fatty acid and cholesterol synthesis, both of which were shown by Illman \& Topping (1985) to be enhanced in the liver when feeding soluble oat fibre, the former by $400 \%$, the latter by $100 \%$. The increase in cholesterol synthesis is to be expected from the increased steroid excretion caused by the oat fibre. The increase in fatty acid synthesis is evidently due to the increased flow of short-chain fatty acids in the liver. In fact, Illman \& Topping (1985) found a positive correlation $(r 0.74, P<0.01)$ between plasma propionate concentrations and hepatic fatty acid synthesis. Thus, it is possible that a dose of oat fibre exceeding that causing the maximum cholesterol reduction will be reflected in increased accumulation of lipids in the liver. Since neither insoluble fibre (Lopez-Guisa et al. 1988) nor oat-bran concentrates in diets containing no added cholesterol or cholic acid caused any accumulation of lipids in liver (Shinnick et al. 1990, and the present study), and since the induced hypercholesterolaemia is an artificial state dissimilar to human metabolism, it is unlikely that oat-bran concentrates would cause a similar effect in human liver.

The basic difficulty in testing the effects of fibre preparations in animal experiments is the lack of animal species having a cholesterol metabolism similar to that of humans. Although the amounts of cholesterol and cholic acid needed to create a hypercholesterolaemic state are usually 10 and $2 \mathrm{~g} / \mathrm{kg}$ respectively, the fat content of the diets is often only $50-100 \mathrm{~g} / \mathrm{kg}$ (Lopez-Guisa et al. 1988; Shinnick et al. 1988, 1990; Ranhotra et al. 1990; Mälkki et al. 1992; Newman et al. 1992), which causes an abnormally high concentration of cholesterol and cholic acid in the fat phase of the feed. A possible way to eliminate these abnormalities is to use young rats which react to the soluble-fibre preparations without needing added lipotropic compounds in the diet (Ranhotra et al. 1990; Mälkki et al. 1992). 
Autio, K., Mälkki, Y., Virtanen, T. (1992). Effects of processing on the microstructure of oat (Avena sativa) bran concentrate and the physicochemical properties of isolated $\beta$-glucans. Food Structure 11, 47-54.

Carrol, R. M. \& Feldman, E. B. (1989). Lipids and lipoproteins. In The Clinical Chemistry of Laboratory Animals, pp. 95-116 [W. F. Loeb and F. W. Quimby, editors]. Oxford: Pergamon Press.

The Committee on Enzymes, The Scandinavian Society for Clinical Chemistry and Clinical Physiology (1974). Recommended methods for the determination of four enzymes in blood. Scandinavian Journal of Clinical Laboratory Investigations 33, 291-306.

Craig, J. R. (1990). Liver. In Anderson's Pathology, 9th ed., pp. 1199-1320 [J. M. Kissane, editor]. St Louis, MO: The C. V. Mosby Co.

Illman, R. J. \& Topping, D. L. (1985). Effects of dietary oat bran on faecal steroid excretion, plasma volatile fatty acids and lipid synthesis in rats. Nutrition Research 5, 839-846.

Lopez-Guisa, J. M., Harned, M. C., Dubelzieg, R., Rao, S. C. \& Marlett, J. A. (1988). Processed oat hulls as potential dietary fiber sources in rats. Journal of Nutrition 118, 953-962.

McCleary, B. \& Glennie-Holmes, M. (1985). Enzymic quantification of $(1 \rightarrow 3)(1 \rightarrow 4)$ - $\beta$-D-glucan in barley and malt. Journal of the Institute of Brewing 91, 285-295.

Mälkki, Y., Autio, K., Hänninen, O., Myllymäki, O., Pelkonen, K., Suortti, T. \& Törrönen, R. (1992). Oat bran concentrates: physical properties of $\beta$-glucan and hypocholesterolemic effects in rats. Cereal Chemistry 69 , $647-653$.

Mongeau, R., Brassard, R., Malcom, S. \& Shah, B. G. (1991). Effect of dietary cereal brans on body weight and blood lipids in a long-term rat experiment. Cereal Chemistry 68, 448-453.

National Research Council (1987). Nutrient requirements of laboratory animals. In Nutrient Requirements of Domestic Animals, p. 23. Washington, DC: National Academy of Sciences.

Newman, R. K., Klopfenstein, C. F., Newman, C. W., Guritno, N. \& Hofer, P. J. (1992). Comparison of the cholesterol-lowering properties of whole barley, oat bran, and wheat red dog in chicks and rats. Cereal Chemistry 69, 240-244.

Ney, D. M., Lasekan, J. B. \& Shinnick, F. L. (1988). Soluble oat fiber tends to normalize lipoprotein composition in cholesterol-fed rats. Journal of Nutrition 118, 1455-1462.

Paton, D. (1991). Processes for the preparation of $\beta$-glucan enriched oat bran. In Proceedings of the International Conference on Oat $\beta$-Glucans and Cardiovascular Disease. Minneapolis, MN: American Oat Association.

Ranhotra, G. S., Gelroth, J. A., Astroth, K. \& Rao, C. S. (1990). Relative lipidemic responses in rats fed oat bran or oat bran concentrate. Cereal Chemistry 67, 509-511.

Schmidt, E., Schmidt, F. W., Both, R., Ohlendorf, S., Raupach, R. \& Staar, U. (1983). Enzyme pattern in liver and serum of rats during growing and aging. In Progress in Clinical Enzymology, vol. 2, pp. 43-52 [D. Goldberg and M. Werner, editors]. New York: Masson.

Shinnick, F. L.., Ink, S. L. \& Marlett, J. A. (1990). Dose response to a dietary oat bran fraction in cholesterol-fed rats. Journal of Nutrition $120,561-568$.

Shinnick, F. L., Longacre, M. J., Ink, S. L. \& Marlett, J. A. (1988). Oat fiber: composition versus physiological function in rats. Journal of Nutrition 118, 144-151. 


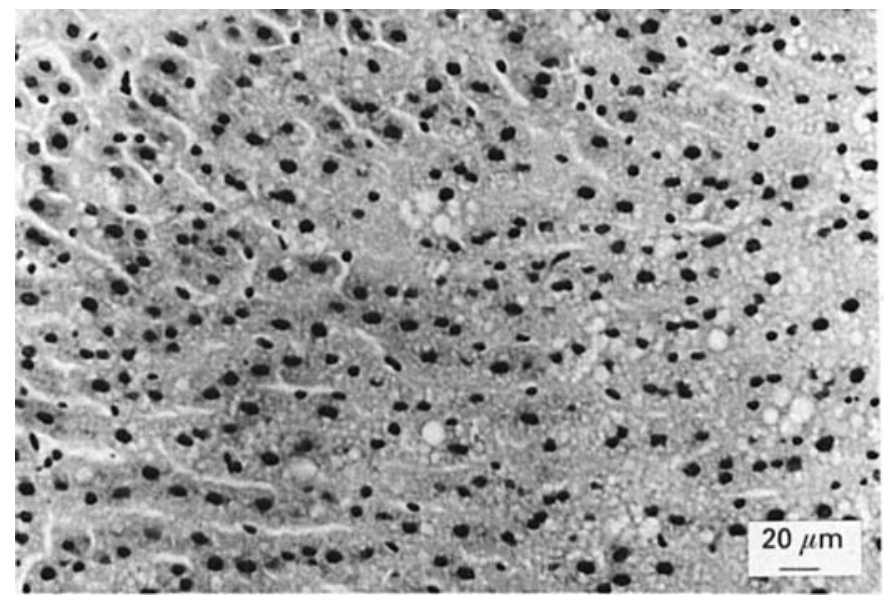

Plate 1. An extensive microvacuolar fatty degeneration (type I, grade $4+$ ) in hypercholesterolaemic rats fed on diets containing oat-bran concentrate. Almost all the parenchymal cells contain multiple small vacuoles in their cytoplasm. None of the cells in this field are severely affected, however. Haematoxylin-eosin. For details of procedures and classification, see p. 769 and Table 3.

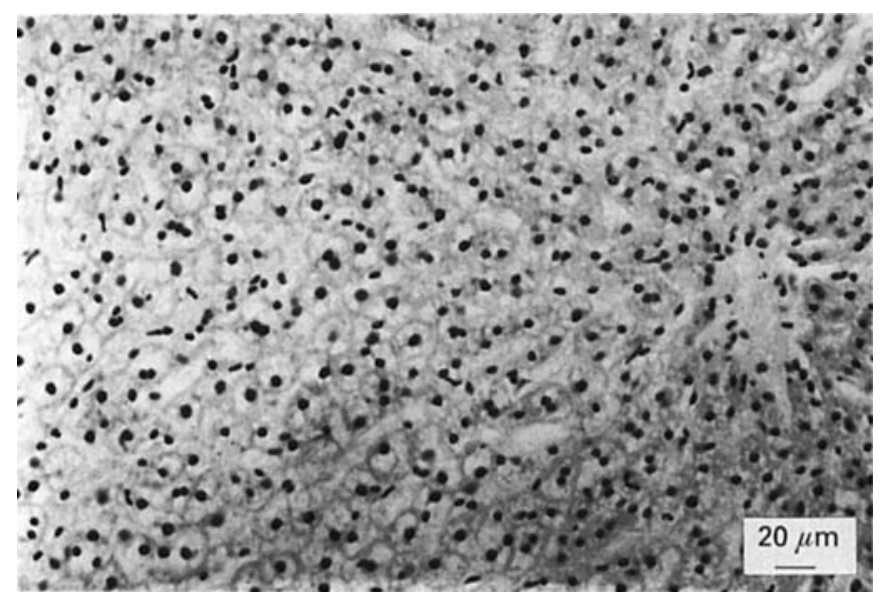

Plate 2. A moderate degree of microvacuolar fatty degeneration (type I, grade $3+$ ) of hypercholesterolaemic rats fed on diets containing oat-bran concentrate. About $75 \%$ of the cells are characterized by extensive cytoplasmic vacuolization. Notice an area of less affected cells around the central vein on the right. Haematoxylin-eosin. For details of procedures and classification, see p. 769 and Table 3. 


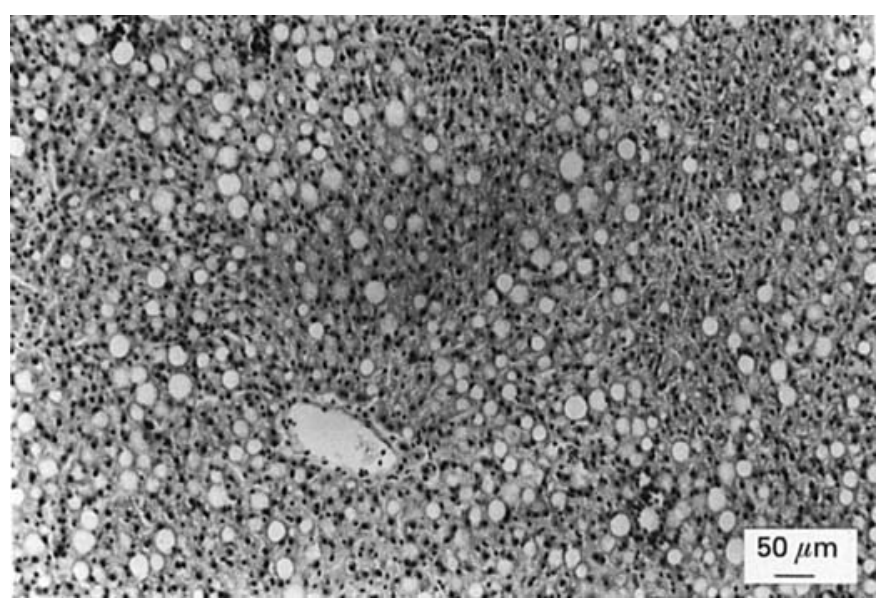

Plate 3. A low magnification view of a severe fatty degeneration (type II, grade 4) of hypercholesterolaemic rats fed on diets containing oat-bran concentrate. More than $75 \%$ of the parenchymal cells contain a single large cytoplasmic vacuole displacing the nucleus to the cell periphery. The remainder of the parenchymal cells are affected by a microvacuolar (mild) fatty degeneration. Haematoxylin-eosin. For details of procedures and classification, see p. 769 and Table 3. 